\title{
SINTOMAS DEPRESSIVOS, ESTRATÉGIAS DE APRENDIZAGEM E RENDIMENTO ESCOLAR DE ALUNOS DO ENSINO FUNDAMENTAL ${ }^{1}$
}

\author{
Miriam Cruvinel \\ Evely Boruchovitch
}

\begin{abstract}
RESUMO. Tendo-se em vista que a incidência de sintomas depressivos tem aumentado e conhecendo os efeitos negativos desses sintomas na aprendizagem, o presente estudo tem como objetivo avaliar a relação entre sintomas de depressão e rendimento escolar e estratégias de aprendizagem de alunos do ensino fundamental. Participaram desta pesquisa 169 alunos de $3^{\mathrm{a}}, 4^{\mathrm{a}}$ e $5^{\mathrm{a}}$ séries de uma escola pública da cidade de Campinas. Os alunos eram, em sua maioria, não-repetentes, de ambos os sexos e com idade variando de 8 a 15 anos. Os resultados sugeriram a existência de diferenças significativas entre rendimento escolar em matemática e sintomas depressivos, bem como entre rendimento escolar tanto em português quanto em matemática e o repertório de estratégias de aprendizagem dos participantes. Uma correlação significativa e negativa foi também encontrada entre os sintomas de depressão e o repertório de estratégias de aprendizagem dos participantes.
\end{abstract}

Palavras-chave: depressão infantil, estratégia de aprendizagem, rendimento escolar.

\section{DEPRESSIVE SYMPTOMS, LEARNING STRATEGIES AND ACADEMIC ACHIEVEMENT AMONG ELEMENTARY SCHOOL STUDENTS}

\begin{abstract}
The incidence of depressive symptoms in children has been increasing. It has been demonstrated that these symptoms have negative effects in the learning process. In line with that, the present study has the objective to evaluate the relationship between depressive symptoms, learning strategies and students' academic achievement. The sample was composed of 169 students of $3^{\text {rd }}$, $4^{\text {th }}$ and $5^{\text {th }}$ grades, and from both sexes of a public school in the city of Campinas. Age range varied from 8 to 15 , and the majority of the students had not repeated a school grade level. Results suggested significant differences between depressive symptoms and mathematics achievement, as well as between learning strategies and school achievement. A significant and negative correlation was found between participants' depressive symptoms and their learning strategies repertoire.
\end{abstract}

Key words: infantile depression, learning strategies, school achievement.

Durante muito tempo, pensou-se que a depressão em crianças não existia ,ou então seria muito rara, nessa população. No entanto, a partir da década de 1960 alguns estudos foram realizados e atualmente não há dúvida quanto à ocorrência de depressão na infância (Bandim, Sougey \& Carvalho, 1995; Baptista \& Golfeto, 2000; Andriola \& Cavalcante, 1999; White, 1989; Soares, 2003).

A depressão é considerada um transtorno do humor, uma vez que, do ponto de vista psicopatológico, a alteração e perturbação do humor ou do afeto consiste em um dos mais importantes sintomas depressivos (DSM IV, 1994; White, 1989). O termo depressão tem sido utilizado de forma genérica e muitas vezes distorcida. Além de a depressão envolver fatores afetivos, apresenta também componentes cognitivos, comportamentais, motivacionais e fisiológicos. Na criança, a literatura sugere também a presença de tais alterações, e o que se percebe é que na infância a depressão normalmente vem associada a outras dificuldades, principalmente problemas de comportamento e problemas escolares,

1 Apoio Capes e Cnpq.

* Psicóloga, doutoranda em Educação pela Unicamp, mestre em Psicologia, Desenvolvimento Humano e Educação pela Faculdade de Educação da Unicamp.

\# Psicóloga pela Universidade do Estado do Rio de Janeiro, Ph.D em Educação pela University of Southern California, Los Angeles, Professora do Departamento de Psicologia Educacional da Faculdade de Educação da Universidade Estadual de Campinas-Unicamp, Membro do Grupo de Estudos e Pesquisas em Psicopedagogia (Gepesp) da Unicamp. 
ocasionando um prejuízo no funcionamento psicossocial (White, 1989; Cole, 1990; Harrington, 1993 Anderson \& McGree, 1994).

A associação entre depressão infantil e rendimento escolar tem sido avaliada por alguns autores. Esses estudos revelam que a incidência de depressão parece aumentar entre as crianças com problemas escolares (Soares, 2003; WrightStrawderman \& Watson, 1992; Stevenson \& Romney 1984; Hall \& Haws, 1989; Colbert, Newman, Ney \& Young, 1992; Feshbach \& Feshbach 1987; Pérez \& Urquijo 2001; Weinberg, Mclean, Snider, Nuckols, Erwin \& Brumback, 1989; Goldstein, Paul \& Cohn, 1985; Palladino, Poli, Mais \& Marcheschi, 2000). A dificuldade da família e dos educadores em reconhecer os sintomas de depressão na criança agrava essa situação, pois, muitas vezes, o professor não identifica corretamente esses sintomas em seus alunos e estes acabam não recebendo orientação e tratamento adequados.

Embora as crianças com sintomas depressivos apresentem dificuldades escolares, alguns autores (Colbert e cols. 1992; Brumback, Jackoway \& Weinberg, 1980; Mokros, Poznanski \& Merrick, 1989) têm observado que essas crianças são capazes intelectualmente, não apresentando nenhum déficit de inteligência. Essa constatação sugere que o baixo rendimento pode ser conseqüência da depressão, em função da falta de interesse e motivação da criança em participar de atividades escolares, bem como sua tendência para sentimento de autodesvalorização (Brumback e cols. 1980).

Assim sendo, o presente estudo tem como objetivo avaliar a relação entre sintomas de depressão e rendimento escolar e estratégias de aprendizagem de alunos do ensino fundamental de uma escola pública da cidade de Campinas, São Paulo. Cabe ressaltar que no presente estudo serão investigados os sintomas depressivos isolados.

\section{A INCIDÊNCIA DA DEPRESSÃO NA INFÂNCIA}

Estudos revelam uma grande diversidade nas taxas de incidência de sintomas depressivos em crianças brasileiras e de outros países. No Brasil, os dados sugerem que algumas cidades parecem apresentar uma incidência maior, talvez em função de diferenças socioeconômicas e características locais ou regionais. Uma alta incidência tem sido observada no Recife, na Paraíba e em São Paulo. Bandim e cols. (1995) encontraram 12,5\% de crianças com depressão maior no Recife. Barbosa e Gaião (2001) revelaram $22 \%$ de sintomas depressivos em crianças da Paraíba.
Curatolo (2001) obteve $21 \%$ de crianças com sintomatologia depressiva em São Paulo. Uma pequena incidência de escolares com sintomas depressivos foi observada em outras cidades brasileiras. Soares (2003) observou que 2,3\% dos alunos da $4^{\text {a }}$ série do Sul do país apresentavam sintomas de depressão. Na cidade de Campinas, interior de São Paulo, foi encontrada uma incidência de $3,5 \%$ de crianças com sintomas de depressão (Cruvinel, 2003; Cruvinel \& Boruchovitch, 2003). Baptista e Golfeto (2000) revelaram uma taxa ainda menor de sintomas depressivos em crianças de 7 a 14 anos na cidade de Ribeirão Preto $(1,48 \%)$, enquanto Hallak (2001) verificou que 6\% dos participantes de seu estudo, também de Ribeirão Preto, apresentaram sintomas de depressão.

A literatura internacional também mostra variações nas taxas de incidência. Stevenson e Romney (1984) encontraram 14\% de crianças com sintomas de depressão, Kazdin e French (1983) observaram uma frequiência de 15\%, Kashani, Cantwell e Sheikim (1983) de $13 \%$ e Kaslow, Rehm e Siegel (1984) identificaram 33\% de depressivos. As pesquisas sugerem que de 6 a $7 \%$ de adolescentes e $1,8 \%$ de crianças entre 7 e 12 anos apresentam sintomas depressivos (German e cols., 1987; Leerhsen e cols. 1987, citados por Wright-Strawderman \& Watson, 1992).

Segundo Poznanski e Mokros (1994), são diversos os fatores que contribuem para essa variabilidade. De acordo com os autores, é necessário considerar, nesses estudos, as diferenças na população estudada (população normal x população clínica), os diferentes métodos de avaliação (entrevista clínica $\mathrm{x}$ questionários ou inventários de auto-avaliação), a definição de depressão (sintoma $x$ síndrome) e métodos para definir a evidência de depressão (critérios de diagnóstico e ponto de corte). Baptista e Golfeto (2000) lembram ainda que, além desses fatores, as diferenças regionais, econômicas e culturais na população brasileira podem contribuir para a diversidade dos resultados encontrados.

É importante ressaltar que a variação nas taxas de prevalência também pode ocorrer devido a uma dificuldade relacionada aos critérios de diagnóstico empregados no estudo. Atualmente existe muita controvérsia quanto ao diagnóstico da depressão na infância. Por exemplo, Rehm e Sharp (1999) afirmam que a depressão em crianças se manifesta de uma forma diferente da depressão no adulto, de forma que rejeitam a idéia de que a depressão infantil seja diagnosticada pelos mesmos critérios utilizados para o adulto. 
De acordo com o Manual Diagnóstico e Estatístico de Transtornos Mentais - DSM IV (1994), os sintomas de um quadro de depressão maior são: humor deprimido na maior parte do dia, falta de interesse nas atividades diárias, alteração de sono e apetite, falta de energia, alteração na atividade motora, sentimento de inutilidade, dificuldade para se concentrar, pensamentos ou tentativas de suicídio. Para o diagnóstico de um episódio depressivo maior é necessário que o indivíduo apresente pelo menos cinco dos sintomas citados, em um período de pelo menos duas semanas, sendo que um dos sintomas deve ser o humor deprimido ou falta de interesse. Conforme ainda o DSM, não há diferença entre a depressão no adulto e na criança, embora o profissional devesse levar em conta algumas variações, devidas à idade e fases de desenvolvimento em que a pessoa se encontra.

\section{SINTOMAS DE DEPRESSÃO E ESTRATÉGIAS DE APRENDIZAGEM.}

Além de a depressão infantil interferir no rendimento escolar, algumas investigações têm mostrado que os sintomas depressivos também afetam os hábitos de estudos dos alunos (Palladino e cols. 2001; Pekrun, Goetz, Titz \& Perry, 2002). As técnicas ou métodos que os alunos usam para adquirir uma informação são definidos como estratégias de aprendizagem (Dembo, 1994, citado por Boruchovitch, 1999a). Pozo (1996), baseando-se na definição de Nisbett, Schucksmith e Dansereau (1987), reafirma que estratégias de aprendizagem são procedimentos e atividades utilizados com o objetivo de facilitar a aquisição, o armazenamento e a utilização da informação.

Existem diversas classificações e diferentes tipos de estratégias de aprendizagem. Todavia, a literatura vem empregando o termo estratégia de aprendizagem para designar tanto as estratégias de aprendizagem cognitivas quanto as estratégias metacognitivas. Segundo Dembo, 1994, citado por Boruchovitch (1999a), as estratégias cognitivas são ensaio (repetir, copiar, sublinhar), elaboração (parafrasear, resumir, anotar e criar analogias) e organização (selecionar idéias, usar roteiros e mapas). As estratégias metacognitivas referem-se ao planejamento (estabelecer metas), monitoramento (autotestagem, atenção, compreensão e uso de estratégias) e regulação (ajustar velocidade, reler, rever, uso de estratégias, ajustar ambiente).

A literatura mostra que as estratégias de aprendizagem, apesar de serem extremamente relevantes para a aprendizagem de qualidade, não são suficientes para o sucesso acadêmico, já que diversas variáveis psicológicas e motivacionais, como atribuições de causalidade para sucesso e fracasso escolar, autoconceito, crenças de auto-eficácia, motivação, ansiedade, entre outros, são fatores determinantes no uso efetivo dessas estratégias (McCormick, Miller \& Pressley, 1989). O fator psicológico tem se revelado tão importante que, nas intervenções em estratégias de aprendizagem, acabam recebendo uma atenção especial. Tem sido sugerido que o ensino de estratégias cognitivas e metacognitivas seja acompanhado pelo ensino de estratégias afetivas, visando acentuar a motivação do aluno, bem como modificar variáveis psicológicas e motivacionais que são incompatíveis com o uso eficiente destas estratégias (Boruchovitch, 1994; Hattie, Biggs \& Purdie, 1996).

Os estudos que visam à relação entre sintomas depressivos e o uso de estratégias de aprendizagem são escassos, portanto, pouco se conhece sobre como os sintomas depressivos interferem no uso das estratégias cognitivas e metacognitivas. No entanto, algumas investigações têm sido realizadas acerca da habilidade de memória nas crianças com depressão, a fim de verificar até que ponto a presença de sintomatologia depressiva interfere nessa função cognitiva (Bandim, Roazzi, Sougey, Botelho \& Carvalho, 1998 e Lauer e cols. 1994). Os estudos de Lauer e cols. (1994) e Bandim e cols. (1998) apresentaram resultados semelhantes. Apesar de diferenças metodológicas, os autores verificaram que a dificuldade de memória está diretamente relacionada à severidade da depressão, ou seja, o prejuízo da memória varia de acordo com a gravidade da depressão. Quanto às estratégias de aprendizagem mais especificamente, o estudo de Palladino e cols. (2000) revelou uma forte correlação entre os componentes metacognitivos (estratégias, monitoramento e atribuições) e componentes afetivomotivacionais, entre eles a depressão.

É extremamente importante conhecer as estratégias de aprendizagem dos alunos, bem como saber até que ponto os fatores emocionais, mais especificamente a depressão, podem interferir no uso dessas estratégias, pois, como descrevem Costa e Boruchovitch (2000a), variáveis afetivas podem ser modificadas mediante a ação de programas de intervenção em estratégias de aprendizagem, de forma a favorecer o aproveitamento escolar do estudante. Costa e Boruchovitch (2000a) apontam ainda para a necessidade de um aumento no número de pesquisas brasileiras acerca das estratégias de aprendizagem e 
variáveis que interferem no seu uso. De maneira geral, atualmente, as pesquisas sugerem que há relação entre as variáveis psicológicas e as estratégias de aprendizagem, mas ainda se tem pouca informação sobre a interferência dos sintomas depressivos no repertório e na utilização dessas estratégias.

\section{MÉTODO}

\section{Participantes}

O estudo foi desenvolvido em uma escola municipal da cidade de Campinas, São Paulo, que atendia uma população de nível socioeconômico baixo. Foi entregue a todos os alunos de $3^{\mathrm{a}}, 4^{\mathrm{a}}$, e $5^{\mathrm{a}}$ séries um termo de autorização, solicitando o consentimento dos pais para a participação dos filhos na presente pesquisa. Participaram do estudo somente os alunos cujos pais autorizaram. Assim, a amostra foi composta de 169 crianças de $3^{\mathrm{a}}$ série $(\mathrm{N}=69), 4^{\mathrm{a}}$ série $(\mathrm{N}=69)$ e de $5^{\mathrm{a}}$ série $(\mathrm{N}=31)$ do ensino fundamental. Os alunos eram de ambos os sexos, sendo que $58 \%$ eram do sexo masculino e $41 \%$ do sexo feminino. A faixa etária dos participantes variou de 8 a 15 anos, sendo $43,8 \%$ entre 8 a 9 anos, $45 \%$ entre 10 a 11 anos e $11,2 \%$ entre 12 a 15 anos de idade. Quanto à história escolar, a maioria dos alunos $(73,4 \%)$ não era repetente e $26,6 \%$ da amostra eram repetentes.

\section{Procedimento}

Após o consentimento e aceitação da escola, como cuidado ético, foi solicitada aos pais ou responsáveis pelas crianças uma autorização por escrito para a participação das mesmas na pesquisa. Foi enviado aos pais um termo de autorização, esclarecendo a forma de participação. Esta carta foi encaminhada por intermédio do próprio aluno. $\mathrm{Na}$ carta, os pais foram orientados a respeito dos objetivos do estudo, do tipo de participação requerido, bem como da ausência de prejuízos decorrentes da não-participação. Os estudantes que entregaram o termo de autorização cujos pais permitiram sua participação na pesquisa foram convocados para a coleta de dados, que ocorreu em uma única entrevista. Aos participantes foi assegurado o caráter confidencial do estudo e foi informado que suas respostas não influenciariam de forma alguma suas notas ou desempenho na escola. Aqueles alunos cujos pais não assinaram a autorização ou aqueles que não entregaram o termo de autorização não participaram do estudo. Cabe ressaltar que $8,8 \%$ dos pais não permitiram que seus filhos fossem entrevistados e 16,4 \% não entregaram ao pesquisador $\mathrm{o}$ termo de consentimento devidamente assinado. Toda a coleta de dados foi precedida de um estudo piloto que permitiu concluir a adequação dos instrumentos, bem como dos procedimentos de coleta de dados.

A aplicação dos instrumentos foi realizada no próprio ambiente escolar, sendo que os instrumentos foram aplicados em pequenos grupos, de no máximo quatro crianças e em apenas uma única sessão, conduzida em horário escolar, na presença da primeira autora.

Inicialmente, estabeleceu-se um rapport com os participantes e em seguida foram colhidas as informações referentes aos dados demográficos. O primeiro instrumento aplicado foi a Escala de Estratégias de aprendizagem para avaliar o uso e freqüência das estratégias, seguida pelo Inventário de Depressão Infantil - CDI. Tanto a Escala de Estratégias de aprendizagem quanto o CDI foram gravados em fita cassete, de forma a minimizar a interferência do pesquisador. As instruções e as questões dos dois instrumentos foram ouvidas pelos participantes. A aplicação dos instrumentos teve duração de aproximadamente 40 a 50 minutos, variando em função das séries e idades dos alunos.

Para avaliar o rendimento escolar dos participantes foram solicitados à direção da escola os conceitos dos alunos nas diferentes disciplinas referentes ao primeiro bimestre do ano letivo.

\section{Instrumentos}

Foram utilizados, nesse estudo, os seguintes instrumentos: Inventário de Depressão Infantil (CDI) e a Escala de Avaliação das Estratégias de aprendizagem.

O Inventário de Depressão Infantil (CDI) desenvolvido por Kovacs (1992) e validado para a população brasileira por Gouveia, Barbosa, Almeida e Gaião (1995) consiste em uma escala de autoavaliação destinada a identificar os sintomas de depressão em pessoas de 7 a 17 anos. Neste trabalho optou-se por utilizar o CDI no rastreamento dos sintomas depressivos, tendo-se em vista sua ampla utilização nos estudos epidemiológicos, bem como as vantagens de ser um instrumento econômico, de fácil aplicação e fácil correção e por ser um instrumento já validado para a população brasileira.

Kovacs (1992) cita diversos estudos em que a confiabilidade do CDI em diferentes amostras foi avaliada e conclui que o coeficiente variou de 0,71 a 0,89 , indicando uma aceitável consistência interna do 
instrumento em questão. Mediante a análise fatorial do CDI, Kovacs (1992) encontrou cinco fatores, moderadamente correlacionados, que explicam a depressão: humor negativo, problemas interpessoais, ineficácia, anedonia e auto-estima negativa.

A versão brasileira do CDI ficou composta por 20 itens distribuídos entre sintomas afetivos, cognitivos e comportamentais da depressão. Ao participante solicita-se que marque com um $\mathrm{X}$ a alternativa que melhor descreve seu modo de pensar, agir ou sentir, devendo assinalar apenas uma alternativa. Cada item ou questão possibilita a escolha de três alternativas de resposta que variam de uma escala de 0 (ausência de sintoma) a 2 pontos (sintoma grave). Como exemplo de um item desse inventário: Eu fico triste de vez em quando; Eu fico triste muitas vezes; Eu estou sempre triste.

No presente estudo, optou-se pela retirada do item 9, de forma que o inventário ficou composto por 19 itens no total. O item 9 trata de uma questão que mede a intenção suicida. Tendo-se em vista que o suicídio em crianças é uma condição pouco freqüente (Cassorla, 1987) e que a depressão pode ou não estar associada ao suicídio (Mendels, 1972), e ainda considerando-se que o suicídio constitui apenas um dos sintomas da depressão, acreditou-se ser mais adequada a retirada dessa questão. Assim como para Weisz, Thurber, Proffitt, Sweeney e Legagnoux (1997), a omissão deste item teve a finalidade de evitar que os participantes pudessem se abalar emocionalmente.

Gouveia e cols. (1995) definiram que o ponto de corte no CDI seria 17 pontos, de forma que a criança que obtiver uma pontuação igual ou superior a 17 pontos deverá merecer atenção, uma vez que tal resultado poderá indicar um provável depressivo. Em função da retirada de uma questão no CDI, no presente estudo, o ponto de corte foi cuidadosamente recalculado, passando para 16.

Utilizou-se também a Escala de Estratégia de Aprendizagem, desenvolvida por Boruchovitch e Santos (2001), com a finalidade de avaliar o repertório de estratégias de aprendizagem cognitivas e metacognitivas de alunos do ensino fundamental. A escala é composta por 40 itens fechados, em forma de escala likert, e uma questão aberta, com objetivo de investigar o uso de estratégias não mencionadas nas questões fechadas.

O aluno possui três alternativas de resposta: sempre, algumas vezes e nunca. Cada alternativa recebe uma pontuação, sendo que a alternativa sempre vale 3 pontos, algumas vezes 2 pontos e nunca 1 ponto, com exceção de algumas questões $(28,30,31$,
$32,35,37,38,39$ e 40) que apresentam essa pontuação invertida, devido à redação dos itens. Nessas questões, a pontuação consiste em: sempre valendo 1 ponto, algumas vezes 2 pontos e nunca valendo 3 pontos. $\mathrm{O}$ valor máximo de pontos a ser obtido na escala é de 120 pontos e o valor mínimo é de 40 pontos. A escala pode ser aplicada tanto individualmente quanto de forma coletiva. A título de ilustração, um exemplo de uma questão dessa escala é: Você repete em voz alta, quando vai lendo um texto, para aprender melhor.

Para averiguar a consistência interna da escala, 443 participantes de $3^{\mathrm{a}}, 4^{\mathrm{a}}$ e $5^{\mathrm{a}}$ séries de escolas públicas do ensino fundamental responderam às questões do instrumento. A avaliação da consistência interna da escala, realizada pelo coeficiente de Cronbach, revelou um Alpha de 0,74, indicando que a Escala de Estratégias de Aprendizagem apresenta aceitáveis índices de consistência interna.

Para analisar o desempenho escolar dos alunos do presente estudo, utilizou-se o conceito de duas disciplinas, Português e Matemático, aferido pela escola, durante o primeiro bimestre do ano letivo. Os conceitos empregados pela escola eram: "O" para ótimo, "B" para bom, "S" satisfatório e "I" para insatisfatório. Além disso, levou-se em conta também a presença e ausência de reprovação escolar.

\section{RESULTADOS}

Os dados deste estudo foram analisados quantitativamente, utilizando-se os procedimentos da estatística descritiva e inferencial.

No que concerne ao rendimento escolar dos alunos, avaliado mediante a análise dos conceitos em duas disciplinas, Português e Matemática, no primeiro bimestre do ano letivo, pôde se constatar que a maioria, 49,7\% dos alunos, apresentava um desempenho considerado satisfatório em Português, $29 \%$ demonstraram um bom desempenho, 14\% tinham desempenho insatisfatório e apenas $5,3 \%$ com ótimo desempenho. Já em Matemática, 39,6\% com desempenho satisfatório, 27,2\% com bom rendimento, $18,9 \%$ dos alunos apresentaram rendimento insatisfatório e $13 \%$ tiveram ótimo rendimento em Matemática. No que diz respeito à repetência, verificou-se que 26,6\% dos participantes já haviam sido reprovados, enquanto $73,4 \%$ nunca haviam repetido nenhuma série anterior.

Dados referentes à média e desvio-padrão do CDI em relação ao rendimento escolar e repetência dos participantes podem ser observados na Tabela 1 . 
Tabela 1: Média e Desvio padrão do CDI em relação ao rendimento escolar e a repetência dos participantes.

\begin{tabular}{|c|c|c|}
\hline & Média CDI & Desvio padrão \\
\hline \multicolumn{3}{|c|}{ Conceito em português } \\
\hline Insatisfatório & 6,72 & 5,07 \\
\hline Satisfatório & 6,92 & 4,10 \\
\hline Bom & 6,20 & 4,63 \\
\hline Ótimo & 4,33 & 3,71 \\
\hline \multicolumn{3}{|c|}{ Conceito em matemática } \\
\hline Insatisfatório & 7,44 & 5,37 \\
\hline Satisfatório & 7,40 & 4,32 \\
\hline Bom & 5,43 & 3,60 \\
\hline Ótimo & 4,91 & 3,87 \\
\hline \multicolumn{3}{|l|}{ Repetência } \\
\hline Repetente & 7,13 & 3,90 \\
\hline Não repetente & 6,29 & 4,54 \\
\hline Total & 6,51 & 4,38 \\
\hline
\end{tabular}

A Análise de Variância (ANOVA) revelou diferenças significativas entre os grupos com diferentes rendimentos em Matemática quanto aos sintomas depressivos $(F=2,688 ; p=0,033)$. Mais precisamente, pôde se observar que alunos com ótimo desempenho em Matemática foram os que apresentaram uma menor média no CDI. O inverso ocorreu entre os alunos que obtiveram conceito insatisfatório em Matemática.

Por outro lado, a Análise de Variância (ANOVA) apontou inexistência de diferenças significativas entre os diferentes grupos de rendimento escolar na disciplina de Português no que se refere aos sintomas depressivos $(F=0,913 ; p=0,458)$. Todavia, foi interessante notar, conforme a Tabela 1 , que os participantes com desempenho satisfatório e insatisfatório nas disciplinas de Português e Matemática apresentaram escores no CDI levemente superiores, quando comparados com os estudantes com bom e ótimo desempenho.

Cabe mencionar que, apesar de os participantes repetentes apresentarem uma média mais alta no CDI do que os não-repetentes, o resultado do Teste $\mathrm{t}$ não revelou diferenças significativas entre esses dois grupos, no que concerne à sintomatologia depressiva.

A Tabela 2 descreve a média e desvio-padrão dos escores dos participantes na Escala de Estratégias de aprendizagem em relação ao rendimento escolar e à repetência.
Tabela 2: Média e desvio-padrão dos escores dos participantes na Escala de Estratégias de Aprendizagem em relação ao rendimento escolar e à repetência.

\begin{tabular}{lcc}
\hline & $\begin{array}{c}\text { Média Escala Estratégia } \\
\text { de aprendizagem }\end{array}$ & Desvio Padrão \\
\hline Conceito em português & 90,88 & 8,39 \\
Insatisfatório & 91,35 & 7,63 \\
Satisfatório & 94,24 & 8,12 \\
Bom & 96,77 & 7,01 \\
Ótimo & & \\
Conceito em & & \\
matemática & 90,90 & 7,78 \\
Insatisfatório & 90,79 & 7,69 \\
Satisfatório & 94,10 & 8,27 \\
Bom & 96,09 & 6,99 \\
Ótimo & & \\
\hline Repetência & 90,66 & 6,94 \\
Repetente & 92,92 & 8,28 \\
Não repetente & $\mathbf{9 2 , 3 2}$ & $\mathbf{7 , 9 9}$ \\
\hline Total & &
\end{tabular}

A Análise de Variância (ANOVA) revelou diferenças significativas no repertório de estratégias de aprendizagem entre os grupos com diferentes rendimentos em Matemática $(\mathrm{F}=3,387 ; \mathrm{p}=0,011) \mathrm{e}$ entre os grupos com diferentes rendimentos em Português $(F=2,550 ; p=0,041)$. Como mostra a Tabela 2, os resultados sugerem que os participantes com ótimo desempenho em Português e Matemática apresentaram uma pontuação mais elevada na Escala de Estratégias de Aprendizagem.

No que diz respeito à repetência, os resultados do Teste $\mathrm{t}$ indicaram não haver diferença significativa entre os dois grupos $(\mathrm{t}=1,773$ e $\mathrm{p}=0,079)$. Entretanto, é interessante notar que os participantes não repetentes revelaram um desempenho levemente superior ao dos repetentes na Escala de Estratégias de Aprendizagem.

Finalmente, a correlação de Pearson indicou uma relação significativa e negativa $(r=-, 207 ; p=0,007)$ entre sintomas depressivos e estratégias de aprendizagem. Os resultados parecem mostrar que os sintomas depressivos tendem a interferir no repertório de estratégias de aprendizagem, ou seja, quanto maior a presença de sintomas depressivos, menor o relato de uso de estratégias de aprendizagem, por parte dos estudantes.

\section{DISCUSSÃO}

O presente estudo teve como objetivo investigar a relação entre os sintomas depressivos, o repertório de 
estratégias de aprendizagem e rendimento escolar de alunos de $3^{\mathrm{a}}, 4^{\mathrm{a}}$ e $5^{\mathrm{a}}$ séries do ensino fundamental de uma escola pública de Campinas.

No que concerne à relação entre sintomas depressivos e rendimento escolar, verificou-se uma relação significativa entre os sintomas depressivos e o rendimento em Matemática, mas por outro lado, não houve relação significativa entre os sintomas depressivos e o rendimento em Português. Este resultado sugere que os sintomas de depressão na criança tendem a interferir mais negativamente no desempenho na disciplina de Matemática do que na disciplina de Português. No entanto, algumas considerações devem ser levantadas quanto a este dado. Por exemplo, o critério empregado para avaliar o rendimento escolar no presente estudo foi 0 conceito/nota dado pelos professores das disciplinas de Matemática e Português, de forma que esses conceitos estão propensos à subjetividade dos professores. A diferença na maneira como cada professor avalia o rendimento escolar de seu aluno pode ser levantada como hipótese para explicar a ausência de relações significativas entre sintomas depressivos e desempenho em Português e a presença de relações significativas entre sintomas depressivos e desempenho em Matemática.

Estudos prévios revelam uma estreita associação entre depressão infantil e rendimento escolar (Soares, 2003; Wright-Strawderman \& Watson, 1992; Stevenson \& Romney 1984; Hall \& Haws, 1989; Colbert e cols. 1992; Pérez \& Urquijo 2001; Weinberg e cols. 1989; Palladino e cols. 2000), no entanto é interessante notar que as investigações anteriores não têm encontrado diferenças significativas quando comparam grupo de deprimidos e grupo de nãodeprimidos quanto ao desempenho em diferentes provas como Aritmética e Português (Brumback e cols. 1980; Pérez \& Urquijo 2001; Tesiny, Lefkowitz \& Gordon, 1980 e Mokros e cols. 1989).

De acordo com Riviere (1995), a dificuldade em Matemática pode ocorrer em função de um problema de atenção ou de uma dificuldade em empregar estratégias adequadas de armazenamento, ou ainda devido à falta de um conhecimento prévio. $\mathrm{O}$ autor reconhece que fatores motivacionais e emocionais podem interferir no desempenho em Matemática. E acrescenta a importância das interações sociais e da comunicação do aluno com um colega de classe ou com professor na aprendizagem da Matemática. Por outro lado, a literatura revela que, na criança com depressão, a memória de curto e memória de longo prazo (Bandim e cols. 1998; Lauer e cols. 1994), a concentração e atenção encontram-se prejudicadas
(Soares, 2003), bem como são mais freqüentes comportamentos como isolamento e falta de interação social (Cole, 1990). Parece que justamente os fatores importantes na aprendizagem de Matemática são aqueles que mais se encontram prejudicados na criança depressiva. Talvez essa explicação permita uma melhor compreensão da relação encontrada no presente estudo entre sintomas de depressão e rendimento em Matemática. Tendo em vista que o resultado encontrado na presente pesquisa diferiu de estudos anteriores, faz-se necessária a realização de mais estudos para que se possa conhecer melhor o impacto da sintomatologia depressiva no rendimento acadêmico, de modo geral e em disciplinas específicas.

No que se refere ao repertório de estratégias de aprendizagem, os resultados do presente estudo sugerem que alunos com ótimo desempenho, tanto em Matemática quanto em Português, são mais estratégicos nas atividades escolares do que os alunos com desempenho satisfatório e insatisfatório nas duas disciplinas. Resultados semelhantes foram obtidos nos estudos de Loranger (1994) e Pokay e Blumenfeld (1990). Estudos brasileiros sobre a relação entre rendimento escolar e estratégias de aprendizagem encontraram diferenças no repertório de estratégias de aprendizagem entre alunos repetentes e não repetentes (Boruchovitch, 1999b e 2001b, Costa, 2000).

$\mathrm{Na}$ presente investigação, os alunos não repetentes mencionaram o emprego de mais estratégias de aprendizagem do que os alunos repetentes. No entanto, cabe questionar se os participantes usam de fato de forma eficiente as estratégias de aprendizagem por eles mencionadas, pois, como mostra a literatura da área, o mais importante não é simplesmente conhecer e utilizar estratégias cognitivas e metacognitivas, mas sim, saber usá-las com eficiência. Portanto, pesquisas futuras devem direcionar seus esforços no sentido de gerar informações acerca da utilização de estratégias de aprendizagem por parte dos alunos, aspecto que, embora muito relevante, não foi objetivo do presente estudo.

Em síntese, a presente pesquisa confirma dados da literatura no sentido de que parece existir uma diferença entre os hábitos de estudos e o uso de estratégias de aprendizagem entre alunos com bom rendimento escolar e alunos com desempenho insatisfatório, principalmente ao nível do conhecimento e do relato das estratégias de aprendizagem por parte dos estudantes.

Outra variável que demonstrou interferir de forma negativa no emprego de estratégias de aprendizagem é 
a presença de sintomas de depressão. Foi encontrada uma correlação significativa e negativa entre a presença de sintomas de depressão e o uso de estratégias de aprendizagem, sugerindo que quanto maior o número de sintomas de depressão, menor o relato de uso de estratégias de aprendizagem pelos participantes.

Resultados semelhantes também foram encontrados em estudos prévios, que confirmaram a idéia de que os fatores afetivos afetam negativamente a aprendizagem do aluno. Palladino e cols. (2000) verificaram que o monitoramento da compreensão e conhecimento estratégico apresenta uma correlação negativa com sentimentos depressivos. Os autores concluíram que o sistema metacognitivo e o sistema afetivo estão relacionados e um influencia o outro, explicando que os estudantes com dificuldade escolar apresentam defícits no sistema metacognitivo. Segundo Dweck (1986), a dificuldade metacognitiva contribui para o desenvolvimento de crenças de desamparo, conseqüentemente conduzindo a sentimentos depressivos (Borkowski, 1992).

\section{CONSIDERAÇÕES FINAIS}

É certo que fatores motivacionais e emocionais como auto-estima (Loranger, 1994), ansiedade (Costa, 2000; Costa \& Boruchovitch 2000a) e sintomas depressivos podem interferir na aprendizagem e no uso eficiente de estratégias de aprendizagem. Costa e Boruchovitch (2000a) enfatizam a importância de algumas dessas variáveis psicológicas e afirmam que fatores motivacionais, emocionais, atribuições de causalidade e ansiedade podem ser modificados mediante intervenção psicopedagógica, quando incompatíveis com o sucesso escolar. O mesmo pode se pensar em relação à sintomatologia depressiva, na qual intervenções poderiam favorecer o uso de estratégias cognitivas e metacognitivas, bem como ensinar estratégias afetivas para lidar melhor com emoções e cognições negativas que acabam atrapalhando o desempenho do aluno com depressão.

Embora o diagnóstico de depressão infantil não seja nem deva ser papel dos educadores, a escola e o professor desempenham uma função extremamente relevante no reconhecimento dos sintomas de depressão, uma vez que a presença da depressão de fato interfere no rendimento do aluno e também tende a influir no emprego de estratégias de aprendizagem. É possível que a queda no rendimento escolar possa ser utilizada como um sinal para os pais e professores de que algo não vai bem com aquela criança e esta pode estar vivenciando sintomas depressivos.
Faz-se necessário que os professores possuam um maior conhecimento e conscientização a respeito do desenvolvimento psicológico infantil, bem como de problemas emocionais comuns nessa faixa etária. Os educadores precisam aprender a reconhecer e identificar os sintomas de depressão em seus alunos e ajudá-los no sentido de diminuir as conseqüências negativas da depressão na aprendizagem. É importante que o professor conheça os efeitos do fracasso escolar na vida psíquica do aluno e adote posturas que previnam tais dificuldades. Na escola é necessário também criar um ambiente que favoreça a aprendizagem, desenvolvendo e aperfeiçoando as estratégias cognitivas e metacognitivas, bem como criar situações que conduzam o aluno a um comportamento de auto-regulação, de forma que ele passe a ter uma postura reflexiva, de autocrítica e de autocontrole perante seus próprios processos cognitivos e afetivos.

Conclui-se pela necessidade de um maior número de pesquisas brasileiras a respeito da influência de variáveis psicológicas na aprendizagem. Futuras pesquisas podem contribuir também para a elaboração de programas de intervenção que visem à prevenção de problemas emocionais na infância, pois, os efeitos de dificuldades psicológicas podem ser duradouros e prejudicar o desenvolvimento infantil. Intervenções conjuntas entre estratégias afetivas e estratégias cognitivas no próprio ambiente escolar são extremamente relevantes.

\section{REFERÊNCIAS}

Anderson, J. C. \& Mcgee, R.. (1994). Comorbidity of Depression in Children and Adolescents. In Reynolds, W. M., Handbook of Depression in Children and Adolescents, (pp.581-602). New York: Plenum Press.

Andriola, W.B. \& Cavalcante, L.R. (1999). Avaliação da depressão infantil em alunos da pré escola. Psicologia: Reflexão e Crítica, 12 (2), 419-428.

Bandim, J.M, Sougey, E.B., \& Carvalho, T.F.R.. (1995). Depressão em crianças: características demográficas e sintomatologia. Jornal Brasileiro de Psiquiatria. 44(1), 27-32.

Bandim, J.M, Roazzi, A., Sougey, E. B. \& Carvalho, T.F.R. (1998). Habilidade mnemônica em crianças com sintomas depressivos: um estudo exploratório. Revista de Neuropsiquiatria na infância e adolescência. 6(3), 119-123.

Baptista, C. A. \& Golfeto, J.H. (2000). Prevalência de depressão em escolares de 7 a 14 anos. Revista de Psiquiatria Clínica. 27(5), 253-255.

Barbosa, G. A. \& Gaião, A.A. (2001). Apontamentos em Psicopatologia infantil. João Pessoa: Idéia.

Borkowski, J. G. (1992). Metacognitive theory: A framework for teachung literacy, writing and math skills. Journal of Learning Disabilities, 25, 253-257. 
Boruchovitch, E. (1993). A Psicologia cognitiva e a metacognição: novas perspectivas para o fracasso escolar brasileiro. Tecnologia Educacional. 22, 22-28.

Boruchovitch, E. (1994). As variáveis psicológicas e o processo de aprendizagem: uma contribuição para a psicologia escolar. Psicologia: Teoria e Pesquisa. 10(1), 129-139.

Boruchovitch, E. (1999a). Estratégias de aprendizagem e desempenho escolar: considerações para a prática educacional. Psicologia: Reflexão e Crítica. 12(2), 361-376.

Boruchovitch, E. (1999b). Developmental Differences in the use of learning strategies among Brasilian students.(p. 76). In Anais do VI European Congress of Psychology, 1999, Roma.

Boruchovitch, E. \& Costa, E. R. (2001). O impacto da ansiedade no rendimento escolar e na motivação de alunos. (pp. 134-147). Em Boruchovitch, E. \& Bzuneck, J.A, (Orgs.) A motivação do aluno. Rio de Janeiro: Vozes.

Boruchovitch, E. (2001a). Inteligência e Motivação: perspectivas atuais. In Boruchovitch, E. \& Bzuneck, J.A, (Orgs.) A motivação do aluno. (pp. 96-115). Rio de Janeiro: Vozes.

Boruchovitch, E. (2001b). Algumas estratégias de compreensão em leitura de alunos do ensino fundamental. Psicologia Escolar $e$ Educacional. 5 (1), 19-25.

Boruchovitch, E., \& Santos, A. A. A. (2001) Escala de Estratégias de Aprendizagem para Alunos do ensino Fundamental. (Manuscrito Não-Publicado).

Brumback, R.A, Jackoway, M.K. \& Weinberg, W.A. (1980). Relation of Intelligence to childhood depression in children referred to Educational Diagnostic Center. Perceptual and Motor Skills. 50, 11-17.

Cassorla, R. M. S. (1987). Comportamentos suicidas na infância e adolescência. Jornal Brasileiro de Psiquiatria. 36(3), 137-144.

Colbert, P., Newman, B., Ney, P., \& Young, J. (1982). Learning Disabilities as a Symptom of Depression in Children. Journal of Learning Disabilities. 15(6), 333-336.

Cole, D. A. (1990). Relation of Social and Academic Competence to Depressive Symptoms in Childhood, Journal of Abnormal Psychology, 99, 422-429.

Costa, E. R. (2000). Estratégias de aprendizagem e a ansiedade de alunos do ensino fundamental: implicações para a prática educacional. Dissertação de Mestrado, Não-Publicada, Pósgraduação em Psicologia, Desenvolvimento Humano e Educação da Faculdade de Educação. Universidade Estadual de Campinal.

Costa, E. R. \& Boruchovitch, E. (2000a). Fatores que influenciam no uso de estratégias de aprendizagem. Psico-USF, 5(1), 11-24.

Costa, E.R., \& Boruchovitch, E. (2000b). Estratégias de Retenção de informações conforme a série e a idade (p. 160). Em Anais do III Congresso Brasileiro de Psicologia do Desenvolvimento, Rio de Janeiro.

Curatolo, E. (2001). Estudo da Sintomatologia depressiva em escolares de sete a doze anos de idade. (Resumo) Em Arquivos de neuropsiquiatria, XVI Congresso Brasileiro de Neurologia $e$ Psiquiatria Infantil. (p. 215) Campinas.

Cruvinel, M. (2003). Depressão infantil, rendimento escolar $e$ estratégias de aprendizagem em alunos do ensino fundamental. Dissertação de Mestrado, Não-Publicada, Pós-graduação em Psicologia, Desenvolvimento Humano e Educação da Faculdade de Educação, Universidade Estadual de Campinas.
Cruvinel, M. \& Boruchovitch, E. (2003). Depressão infantil: uma contribuição para a prática educacional. Psicologia escolar $e$ educacional, 7(1), 77-84.

Dembo, M.H. (1994, $5^{\text {a }}$ ed.). Applyng educational psychology. New York: Longman Publishing Group.

Dweck, C.S. (1986). Motivational processes affecting learning. American Psychology, 41, 1040-1048.

Feshbach, N. D. \& Feshbach, S. (1987). Affective processes and academic achievement. Child Development, 58(5), 1335-1347.

Goldstein, D., Paul, G. G. \& Cohn, S. S. (1985). Depression and achievement in subgroups of children with learning disabilities. Journal of Applied Developmental Psychology. 6(4), pp. 263-275, 1985.

Gouveia, V. V., Barbosa, G.A, Almeida, H. J. F. \& Gaião, A. A. (1995). Inventário de depressão infantil - CDI: Estudo de adaptação com escolares de João Pessoa. Jornal Brasileiro de Psiquiatria, 44, 345-349.

Hall, C. W. \& Haws, D. (1989). Depressive symptomatology in learning-disabled and nonlearning-disabled students. Psychology in the Schools, 26, 359-364.

Hallak, L. R. L. (2001). Estimativa da prevalência de sintomas depressivos em escolares da rede pública de Ribeirão Preto. Ribeirão Preto, Dissertação de Mestrado, Não-Publicada, Pósgraduação em Saúde mental do departamento de Neurologia, Psiquiatria e Psicologia Médica da Faculdade de Medicina de Ribeirão Preto da Universidade de São Paulo.

Harrington, R. (1993). Depressive Disorder in Childhood and Adolescence. New York: John Wiley \& Sons.

Hattie, J., Biggs, J. \& Purdie, N. (1996) Effects of learning skills interventions on students: A meta analysis. Review of Educational Research, 66, 99-136.

Kashani, J., Cantwell, D.P. \& Sheikim, W.O, (1983). Major depressive disorder in children admitted to na inpatient community mental health center. American journal of psychiatry, 139, 671-672.

Kaslow, N. J., Rehm, L.P. \& Siegel, A W., (1984) Social-Cognitive and Cognitive Correlates of Depression in children. Journal Abnormal Child Psychology. 12(4), 605-620.

Kazdin, A E., \& French, N.B. (1983). Assentment of childhood depression: correspondence of child and parent ratings. Journal American Academic Child Psychiatry, 22, 157-164.

Kovacs, M. (1992). Children Depression Inventory CDI: Manual. New York: Multi-Health Systems, Inc.

Lauer, R. E, Giordani, B., Boivin, J. M., Halle, N., Glasgow, B., Alessi, N.E, \& Berent, S., (1994). Effects of depression on memory performance and metamemory in children. Journal American Acad. Child Adolescent Psychiatry, 33, 679-685.

Loranger, A L. (1994). The study strategies of successful and unsuccessful high school students. Journal of Reading Behavior, 26(4), 347-360.

Mccormick, C. B., Miller, G. \& Preesley, M. (1989). Cognitive Strategy research from basic research to educational applications, Springer-Verlaq,

Mendels, J. (1972) Conceitos de depressão. (C. M. Rêgo, Trad.). Rio de Janeiro: Editora Livros técnicos e científicos.

Mokros, H.B., Poznanski, E. O., \& Merrick, W. A. (1989). Depression and Learning Disabilities in Children: A test of an Hypothesis. Journal of Learning Disabilities, 22(4), 230-233. 
Palladino, P., Poli, P., Masi, G., \& Marcheschi, M., (2000). The relation between metacognition and depressive symptoms in preadolescents with learning disabilities: data in support of Borkowski's Model, Learning Disabilities Research \& Practice, 15(3), 142-148.

Pekrun, R., Goetz, T., Titz, W., \& Perry, R.P. (2002). Academic emotions in students' self-regulated learning and achievement: a program of qualitative and quantitative research. Educational Psychologist, 37(2), 91-105.

Pérez, M. V. \& Urquijo, S. (2001). Depresión en adolescentes. Relaciones com el desempenõ académico. Psicologia Escolar $e$ Educacional, 5(1), 49-58.

Pokay, P. \& Blumenfeld, P. C., (1990). Predicting achievement early and late in the semester: the role of motivational and use of learning strategies. Journal of Educational Psychology, 82(1), 4150.

Poznanski, E. O, \& Mokros, H. B. (1994). Phenomenology and epidemiology of mood disorders in children and adolescents. In Reynolds, W. M., Handbook of Depression in Children and Adolescents, Plenum Press, New York, 19-40.

Pozo, J. J., (1996). Estratégias de Aprendizagem. Em: Coll, J. P. \& A Marchesi (Orgs.), Desenvolvimento psicológico e educação: Psicologia da Educação, (pp.176-197). Porto Alegre: Artes Médicas.

Rehm, L. P., \& Sharp, R. N. (1999). Estratégias para a depressão infantil. Em: M. Reinecke, F. Dattilio \& Artur Freeman, Terapia Cognitiva com crianças e adolescentes. (pp.91-104). São Paulo: Artes Médicas.
Soares, M. U, (2003). Estudos das variáveis do desenvolvimento infantil em crianças com e sem o diagnóstico de depressão. Tese de Doutorado Não-Publicada, Pós-graduação em Ciência do Movimento Humano, Universidade Federal de Santa Maria.

Stevenson, D. T. \& Romney, D. M., (1984). Depression in Learning Disabled Children. Journal of Learning Desabilities, 17(10), 579582.

Tesiny, E.P., Lefkowitz, M. M, \& Gordon, N.H. (1980). Childhood Depression, Locus of control, and school achievement. Journal of Educational Psychology. 72(4), 506-510.

Weinberg, W.A, Mclean, A, Snider, R. L., Nuckols, A S., Rintelmann, J. W., Erwin, P. R. \& Brumback, R.A, (1989). Depression, Learning Disability and school behavior problems. Psychological Reports, 64, 275-283.

Weisz, J. R., Thurber, C.A, Proffitt, V. D, Sweeney, L. \& Legagnoux, G. L. (1997). Brief Treatment of Mild-to-moderate child depression using primary and secondary control enhancement training. Journal of consulting and clinical psychology, 65(4), 703-707.

White, J. (1989). Depression. In J., White. The Troubled adolescent. (pp.111-142). Oxford: Pergamon Press.

Wright-Strawderman, C. \& Watson, B.L. (1992). The Prevalence of Depressive Symptoms in Children With Learning Disabilities. Journal of Learning Disabilities, 25(4), 258-264.

Recebido em 23/06/2004 Aceito em 16/09/2004

Endereço para correspondência: Miriam Cruvinel: Rua Antônio Sachi, 351 Chácara da Barra, CEP 13093-070, Campinas-SP. E-mail: miriampsi@hotmail.com 